\title{
Integrating a discovery based remote/hybrid crystallography lab module into an undergraduate teaching laboratory during the pandemic
}

\author{
Joe Tanski \\ Vassar College, Poughkeepsie, New York, United States of America; \\ jotanski@vassar.edu
}

\begin{abstract}
An approach for increasing the impact of undergraduate scientific training with a discovery based X-ray structure determination lab module has been part of the chemistry curriculum at Vassar College since 2010. Just as chemical crystallography and complimentary spectroscopic techniques such as NMR can be fast, effective tools to experimentally determine the structure of molecules and enhance students learning of molecular structure, they can also provide an inspiring opportunity for students to write short, scientific journal style reports that can be edited and published in collaboration with a mentor. This talk will briefly review the X-ray crystallography module and then focus on the experience of conducting this module with remote and hybrid online learning during the pandemic.
\end{abstract}

Keywords: chemical crystallography; education 